\title{
edmetic
}

Revista de Educación Mediática y TIC

\section{Percepción de habilidades docentes a través de las emociones mediante el uso de videojuegos de temática social}

\section{Perception of teaching skills through emotions by the use of social theme video games}

\author{
Jorge Guerra Antequera ${ }^{1}$, María Inmaculada Pedrera Rodríguez ${ }^{2}$ y Francisco Ignacio Revuelta
} Domínguez $^{3}$

Fecha de recepción: 30/05/2018; Fecha de revisión: 17/06/2018; Fecha de aceptación: $24 / 07 / 2018$

\section{Cómo citar este artículo:}

Guerra Antequera, J., Pedrera Rodríguez, Mª I., \& Revuelta Domínguez, F.I., (2018). Percepción de habilidades docentes a través de las emociones mediante el uso de videojuegos de temática social. EDMETIC, Revista de Educación Mediática y TIC, 7(2), 57-77. doi: https://doi.org/10.21071/edmetic.v7i2.10950

\section{Autor de correspondencia; guerra@unex.es}

\section{Resumen}

La consideración del juego como herramienta educativa es una constante en la cultura humana. Actualmente, la esencia del juego es la misma, pero han cambiado los formatos para llevarlo a cabo. En éste marco nacen los videojuegos, los cuáles han tenido una vertiginosa evolución considerándose en los últimos años como elementos educativos mediante el Digital Game Based Learning. También se han tenido en cuenta las emociones como elementos propios de los procesos de aprendizaje vinculados con una serie de atributos y habilidades identificados con la docencia.

La investigación realizada a una muestra $(n=31)$ de estudiantes versa sobre la identificación de aprendizajes de atributos y habilidades docentes y el tratamiento de las emociones a través del análisis de videojuegos con temáticas sociales. Los videojuegos son This War of Mine, Papers, please! y Unmanned. Las técnicas de acopio de información han sido la observación y la entrevista. Mediante éstas técnicas los videojugadores deben identificar qué emociones, atributos y habilidades son capaces de experimentar y/o desarrollar mientras están jugando. Los resultados obtenidos muestran que los sujetos investigados perciben esas emociones y les afecta para la consecución o desarrollo de los atributos y habilidades docentes. A modo de conclusión, se puede señalar que el análisis de los videojuegos fue efectivo y se hallaron respuestas emocionales ante acciones desarrolladas en el juego, así como aprendizajes destacados para el docente del siglo XXI presentes en los tres videojuegos.

Palabras claves: Juego De Ordenador, Formación De Profesores, Análisis Cualitativo, Emoción.

\footnotetext{
1 Universidad de Extremadura, Facultad de Formación del Profesorado (Cáceres, España); guerra@unex.es; ORCID: 0000-0003-1675-8038

2 Universidad de Extremadura, Facultad de Educación (Badajoz, España); inmapedrera@unex.es; ORCID: 0000-0001-5569-2807

3 Universidad de Extremadura, Facultad de Formación del Profesorado (Cáceres, España); fird@unex.es; ORCID: 0000-0002-3649-4327. Autor de correspondencia.
} 


\section{Abstract:}

The consideration of play as an educational tool is a constant in human culture. Currently, the essence of the game is the same, but the formats have changed to make it happen. Within this framework, videogames are born, which have undergone a vertiginous evolution and have been considered in recent years as educational elements through the Digital Game Based Learning. Emotions have also been taken into account as elements of learning processes linked to a series of attributes and skills identified with teaching.

The research carried out on a sample $(n=31)$ deals with the identification of learning attributes and teaching skills and the treatment of emotions through the analysis of video games with social themes. Video games are This War of Mine, Papers, please! and Unmanned. The information gathering techniques have been observation and interview. Using these techniques, gamers must identify what emotions, attributes and skills they are able to experience and/or develop while playing. The results obtained show that the subjects investigated perceive these emotions and are affected for the achievement or development of the teaching attributes and skills. By way of conclusion, it can be pointed out that the analysis of the video games was effective and emotional responses were found to actions developed in the game, as well as outstanding learning for the 21 st century teacher present in the three video games.

Keywords: Computer Game, Teacher Education, Qualitative Analysis, Emotion. 


\section{Introducción}

Las Tecnologías de la Información y la Comunicación (TIC) son una de las herramientas que más rápido han evolucionado a lo largo de la historia. Su inmersión en la educación y su simbiosis en tecnología educativa ha hecho emerger nuevos paradigmas educativos en los que confluyen metodologías y tecnologías. En éste marco surge el paradigma de aprendizaje basado en juegos digitales o Digital Game Based Learning (Prensky, 2001). Este aprendizaje a menudo se orienta sobre las posibilidades educativas de mecánicas de juego, narrativas, metodologías dentro del juego, entre otros. No obstante, desde ésta investigación se pretende además de conocer qué habilidades y competencias se obtienen jugando a un videojuego con una temática social, cuáles son las emociones que emergen y ayudan a entender situaciones, a empatizar o a iniciar un aprendizaje.

El vínculo formado entre educación y videojuegos es un binomio en el que se exploran las posibilidades didácticas implícitas en los videojuegos que la literatura científica considera desde hace años (Gee, 2003; Squire, 2008; McGonigal, 2011; Revuelta y Guerra, 2012; Barr, 2017 y Shanbari y Issa, 2018). No obstante, en éste estudio se incorpora la perspectiva emocional del videojugador ante lo que percibe en el juego. Es decir, se presta atención más allá de los contenidos, concretamente en las dinámicas que vertebran las mecánicas de juego en las que emergen un conjunto de emociones que conllevan la realización de una $u$ otras acciones, realizadas a través de la toma de decisiones. Por ello, en la presente investigación se expone la perspectiva de aproximación al objeto de estudio, la cual promueve la reconfiguración del espacio de observación para adquirir una dinámica emocional en el momento de juego que, en este caso sea el iniciador de implicaciones de corte axiológico entre el vínculo del individuo que juega (videojugador) y los aprendizajes y emociones que se manifiestan en dicha acción. 


\section{Aprendiendo a jugar}

La conjunción del juego con el aprendizaje es una constante antropológica en las diferentes etapas evolutivas de las sociedades que conforman la especie humana. La acción de jugar ha sido inherente al comportamiento humano, no siendo un comportamiento definido en un tiempo o un especio determinado, sino que estaba integrado en las rutinas de vida de nuestros ancestros (Borja, 1980 y Caillois, 1986). El juego es un mecanismo presente en los animales más desarrollados como método para instruir a los ejemplares de más corta edad respecto al desarrollo en el contexto que los circunda (Huizinga, 2000).

En este sentido algunos autores (Huizinga, 2000; Suits, 2005 y Revuelta y Guerra, 2012) señalan que el juego no puede enmarcarse dentro de las acciones humanas racionales, pues no se comprende dentro de las funciones vitales primarias. No obstante, se ha de reflexionar el porqué de éste acto: los animales juegan y desconocen el motivo por el que lo hacen y el ser humano juega y reflexiona sobre el acto de juego a pesar de ser algo irracional y sin un propósito cercano a sentimientos o acciones más viscerales relacionadas con la supervivencia del individuo. Es por ello, que se debe determinar el sentido, la naturaleza del juego en relación con la especie humana para conocer cuál es el papel que desempeña dentro de los mecanismos de aprendizaje propios de la especie humana. Es por ello, que se debe cuestionar al juego como germen de la cultura (Huizinga 2000) una característica propia de nuestra especie.

En este sentido, Revuelta y Guerra (2012) señalan que el ser humano y el juego han ido evolucionando de modo paralelo. Actualmente, el juego sigue existiendo, pero adquiriendo nuevos elementos metodológicos, recursos tecnológicos y narrativas, entre otros, para desembocar en los juegos electrónicos, juegos digitales o videojuegos. Éstos son la herramienta de ocio más popular y extendida. Siguen siendo juegos, simplemente se han incorporado nuevos lenguajes, nuevas mecánicas y nuevas tecnologías, pero la esencia es la misma, la diversión.

Desde ésta consideración surge el paradigma del Aprendizaje Basado en juegos Digitales o Digital Game Based Learning (Prensky, 2001) enmarcado 
dentro del paradigma educativo Edutainment, el cual aboga por ofrecer contenidos didácticos mediante fórmulas asociadas comúnmente al entretenimiento.

Por ello, tal y como señalan Revuelta y Guerra (2012) el docente ha de establecer un vínculo con el discente que le ayude a discernir las necesidades de éste último para contextualizar los conocimientos y herramientas que se ofrecen, lo cual posibilita la inclusión e integración de elementos afines a su realidad percibida postergando a aquellos que no están presentes en su cotidianeidad a planos secundarios. En ésta línea, la inclusión del paradigma DGBL aprovecha el potencial didáctico del producto de ocio más extenso y popular, los videojuegos, ajustando el proceso de enseñanza-aprendizaje a estos instrumentos a priori vinculados con una vertiente lúdica.

En base a ello, Guerra y Revuelta (2016) señalan que los videojuegos están conformados alrededor de una serie de elementos clave que condicionan el uso de éstas herramientas, como son: (a) la narrativa, (b) los gráficos, (c) la música, (d) la experiencia, (e) el estado anímico, (f) la estimulación de la imaginación y (g) la aplicación de estrategias de aprendizaje. Todas ellas están encaminadas a la adquisición de habilidades y/o destrezas así como conocimientos. Dada la interactividad de los videojuegos (Dondlinger, 2007; Klimmt, Hartmann y Frey, 2007; Bruning, Schraw, y Norby, 2011; Revuelta y Guerra, 2012) se toma el contexto como parte del proceso de aprendizaje; situando aquello que se va a aprender en un entorno proclive para lograr una vinculación y motivación inmersiva del jugador con respecto a ese contexto. Es por ello, que el alumnado debe conocer en qué contexto va a desarrollar sus aprendizajes para poder extrapolar lo aprendido de un entorno simulado o virtual al mundo real; sirviendo los videojuegos para el propósito de generar ese espacio de experimentación en el que el discente pueda simular y estructurar las misiones y acatarlas según la situación lo requiera.

En este sentido Tost y Boira (2015) sostienen que un videojuego no sólo está conformado por la estructura audiovisual, sino que en su diseño se han tenido en cuenta las emociones, tanto de las personas involucradas en su 
creación como aquellas que manifiestan las que lo utilizan. Dicha confluencia ha sido fruto de estudios anteriores (Marcano, 2006; González y Blanco, 2008; Del Moral y Fernández (2012) y Guerra y Revuelta (2015) en los que las emociones desempeñaban un papel crucial respecto a la adquisición de aprendizajes mediante el uso de videojuegos, así como un elemento motivador relevante.

No obstante, para conseguir que éste binomio funcione, y la inmersión sea posible, han de alterarse aspectos gráficos, sonoros y narrativos o aquellos relativos a la jugabilidad. Lo que se pretende con ello es hacer que el videojugador ejerza estrategias de reflexión sobre diferentes temáticas, casuísticas o dilemas ético-morales asociando los lenguajes que conforman el juego, vehiculando el aprendizaje sobre las emociones que emergen.

\subsection{Videojuegos analizados}

Para llevar a cabo esta investigación, se han analizado tres videojuegos con un fuerte mensaje ideológico en cuanto a temáticas y con cuestionamientos ético-morales cuyo trasfondo invita a la reflexión y a posicionarse en una perspectiva de controversia sobre las acciones acometidas en los videojuegos. Estos ha sido los videojuegos analizados:

This War of Mine, es un videojuego bélico de acción-aventura del subgénero roguelike ${ }^{4}$ con gráficos $3 \mathrm{D}$ y perspectiva $2.5 \mathrm{D}$ desarrollado por el estudio 11 bits. En éste videojuego el jugador asume el papel de un grupo de personas que luchan por sobrevivir al conflicto que se está desarrollando en un país ficticio llamado Pogoren (inspirado en el conflicto de los Balcanes). Lo novedoso de éste videojuego es que, a pesar de ser un juego de temática bélica, no se asume el papel de un soldado de uno de los bandos contendientes, sino que se asume el papel de una víctima coyuntural del conflicto. El objetivo del juego es mantener el mayor tiempo posible con vida a los personajes. Cuando todos ellos mueren el juego termina.

Respecto de las mecánicas de juego, podemos señalar que se divide en dos partes que representan el ciclo día/noche. Cuando se juega

\footnotetext{
${ }^{4}$ Roguelike: es un género en el que el jugador se adentra en una mazmorra generada de modo aleatorio.
} 
en la fase de día es un juego de gestión de recursos en el que el jugador tendrá que construir, reparar, crear, cocinar, curar y alimentar al grupo de supervivientes. Por otro lado, durante la noche el juego torna sus mecánicas a un juego de exploración y acción en el que jugador debe gestionar al grupo de superviviente para salir al exterior del refugio y buscar víveres, medicinas y materiales de construcción para ser empleados en la fase de día. Las salidas son obligatorias ya que los víveres y los útiles médicos se agotan rápidamente. Los materiales encontrados son utilizados para crear camas, cocinas, mesas de trabajo entre otros, así como, para mejorar el refugio ante enemigos.

No obstante, las salidas realizadas por el grupo son arduas y siempre tienen alguna consecuencia negativa para un personaje o para el grupo. En ellas, el jugador ha de tomar decisiones que regularmente van contra los valores ético-morales, pero han de ser realizadas para asegurar la supervivencia del grupo. Éstas decisiones implican que los personajes tengan que robar comida a familias en su misma situación, tengan que agredir a otros para conseguir medicinas, abandonen a personajes heridos después de robarles, mientan y asesinen. Todas éstas acciones quedarán grabadas no solo en la mente del jugador también en la de los personajes, los cuáles pueden llegar a un nivel de estrés tan alto que les lleven a quitarse la vida.

Papers, please! es un videojuego del género simulación y dentro de éste pertenece al subgénero burocrático. Fue creado por el desarrollador independiente Lucas Pope.

En éste videojuego el jugador asume el papel de un hombre que ha ganado la lotería estatal del ficticio país Arstotzka para obtener un puesto de trabajo, el de burócrata fronterizo. Por ello, el jugador debe gestionar y examinar los documentos de las personas de los países colindantes que quieran cruzar la frontera.

Estéticamente, en cuanto a los gráficos y al sonido recuerda a aquellos juegos de los años 80. Esta estética es intencional ya que se pretende hacer un ejercicio de inmersión mediante un contexto sociopolítico próximo al de los últimos años de Alemania Oriental, 
concretamente durante la finalización de la Perestroika y la caída del Muro de Berlín.

Al igual que en el anterior videojuego, el fin último de éste es el de la supervivencia, no sólo del individuo sino la de su familia entera. Para ello, el jugador tiene que realizar su trabajo de modo exhaustivo sin fallar en lo más mínimo. Cada fallo se penaliza con la retirada de dinero al finalizar el día, lo cual resta en inversión de vivienda, calefacción, medicina y comidas. Así pues, los hechos estarían concatenados y fallar en el trabajo se traduce en pérdida de dinero, la pérdida de éste supone no alimentar calentar o curar a los familiares.

Para llevar a cabo el trabajo en la frontera el jugador dispone de diversas herramientas como manuales, prensa y órdenes gubernamentales, los cuáles irán cambiando a lo largo del juego por lo que se debe prestar atención a las noticias.

En éste sentido, podemos señalar que el videojuego permite que el videojugador empatice con la casuística de las personas que están cruzando la frontera por diversos motivos; posicionándole ante una perspectiva dualista en la que podría ayudar a otras personas frente a las injusticias del sistema a costa de su sueldo y por tanto su supervivencia. 0 ignorar sus principios ético-morales y seguir ganando dinero para mantener a la familia.

Unmanned es un juego serio, es decir, un juego creado con un propósito formativo-instructivo, alejado de la concepción lúdica. Fue creado por el desarrollador independiente creado por Jim Munroe.

Atendiendo a la estética de éste juego es diferente a los dos anteriores presenta un diseño colorido y cercano a las series de dibujos para niños. Su sonido es contextual y ayuda a sumergir al jugador en la mente del personaje principal de la narrativa, aluden a la angustia y el desasosiego que siente. La narrativa es totalmente dicotómica respecto a la estética; reflejando dilemas ético-morales a través de un contexto pseudo-belicista.

En Unmanned el jugador se convierte en un piloto de un vehículo aéreo no tripulado (UAV -Unmanned Aerial Vehicle), es decir, un drone militar que realiza misiones de bombardeo en países de oriente próximo. 
La meta del juego es vivir junto al protagonista la cotidianeidad tanto de su trabajo como la de su faceta de padre y marido. Durante el desarrollo del juego, el protagonista expone al jugador, quien podría ser un ser omnisciente, sus pensamientos y reflexiones sobre las acciones que está llevando a cabo.

La mecánica del juego es la de una historia interactiva, dividida en dos partes. La vida como civil y la vida como militar. Como civil el jugador ha de realizar acciones como afeitar al personaje, jugar con el hijo o dormir. Como militar se han de realizar misiones de vigilancia y eliminación de objetivos. Desde una perspectiva holística el juego ofrece una reflexión compleja sobre el deber y la ética donde la vida posee el valor que el jugador le otorgue.

\subsection{Emociones percibidas}

En éste estudio se ha tomado como referencia la obra de Bisquerra y Punset (2015) en el cuál se señala que las emociones son las respuestas que ofrece el organismo ante la percepción de situaciones de un modo personal e inmaterial, lo cual difiere de la sociedad actual basada en lo material. En base a ello, cabe cuestionarse la naturaleza y el número de emociones existentes, así como el funcionamiento y la vinculación presentes en éstas; surgiendo el concepto del universo de las emociones, proporcionando una perspectiva holística de las emociones existentes y los procesos que engloban. Éste concepto ofrece una visión precisa de cada una de ellas divididas en diferentes galaxias de emociones, las cuáles contienen emociones con características afines.

La consideración que se hace de las emociones se basa en la necesidad de éstas para afrontar las situaciones; clasificándolas en positivas y negativas, aunque no hacen referencia a lo bueno en el caso de las positivas ni a lo malo en las negativas, sino que se utiliza para dotarlas de sentido de bienestar o malestar. Vinculadas con los videojuegos Tost y Boira (2015) inciden en la importancia de las emociones en la interacción entre el videojuego y el videojugador debido a que éstas pueden ayudar al jugador a comprender las emociones 
experimentándolas en una entrono virtual para exportarlas a situaciones en el mundo real pero gestionadas de un modo racional. Tal y como señala McGonigal (2011) la simbiosis que surge de conjugar el contexto emocional y el lúdico para lograr una convergencia. Por lo que se puede señalar que los videojuegos permiten experimentar aprendizajes y/o emociones en un campo de pruebas virtual (Pedrera y González, 2017). Mediante el cual se pretende vehicular el uso del paradigma de aprendizaje basado en juegos con las emociones para que el videojugador sea capaz de poner en prácticas las habilidades adquiridas en el entorno virtual.

\subsection{Adquisición del habilidades y atributos del docente para el siglo XXI}

La utilización de los videojuegos de modo didáctico en conjunción con las emociones, representa la adaptación del docente a los nuevos medios tecnológicos y las demandas sociales a través de la consideración de las emociones como elemento presente en los procesos de enseñanza y aprendizaje. Dicha conjunción se enmarca en la corriente iniciada por Mayer y Salovey (1997) en la que las emociones, y el control de éstas son determinantes para la adquisición de aprendizajes, habilidades y destrezas. Dada ésta vertiginosa evolución tecnológica, social y cultural se hace necesario que el docente adquiera nuevas habilidades y atributos para adecuarse a los nuevos medios, metodologías y configuraciones sociales. Gerstein (2015) propone los siguientes:

Comunicación Oral y escrita: fomentar la expresión oral y escrita entre los estudiantes. Focalizando en el uso de sus ideas y argumentaciones.

Pensamiento crítico y resolución de problemas: fomentar la capacidad analítica mediante el ensayo y el error.

Redes de colaboración: establecer patrones de liderazgos logrados a través de la influencia del trabajo no por autoridad.

Curiosidad e imaginación: agrega un elemento creativo a tus ideas o las de otros.

Iniciativa y emprendimiento: promocionar personas capaces de resolver problema de modo creativo. 
Agilidad y adaptabilidad: capacidad de adaptación a los cambios sociales, culturales y tecnológicos que se presenten de un modo rápido y eficaz.

Optimismo y esperanza: fomentar actitudes positivas y la conceptualización de objetivos.

Autoregulación: aprender a controlar las acciones, pensamientos y emociones de sí mismo como medio para evitar la conflictividad social y las emociones negativas.

Empatía y administración global: promover la consideración de las ideas de los otros respecto a las del uno mismo.

Resiliencia: aprender a afrontar situaciones difíciles aprendiendo de ellas.

Esfuerzo: perseverar en la consecución de una meta.

Visión de futuro: tomar una posición proactiva respecto a las metas personales y profesionales que deseen alcanzar.

\section{Materiales y métodos}

\subsection{Objetivos de investigación}

Los objetivos que pretendemos alcanzar con este estudio son los siguientes:

Analizar los videojuegos seleccionados e indagar sobre los patrones emocionales, habilidades y estrategias propias de los docentes del siglo XXI.

Los objetivos específicos son

Elaborar una lista de con las emociones halladas en los videojuegos analizados.

Crear un mapa de los vínculos entre el desarrollo de atributos y habilidades y los videojuegos analizados.

\subsection{Muestra}

La muestra de éste estudio es de $n=31$, compuesta por 17 chicas $(54,8 \%)$ y 14 chicos $(45,2 \%)$. Las edades están comprendidas entre los 18 y los 22 años. Todo ellos estudiantes del Grado de Educación Primaria de la Facultad de Formación del Profesorado de la Universidad de Extremadura 


\subsection{Procedimiento}

El procedimiento utilizado para esta investigación ha consistido en ubicar al alumnado en una sala de juegos (Laboratorio de Videojuegos y Educación "NodoPlay") provista de los videojuegos señalados anteriormente. Se somete al sujeto a un contacto con el videojuego y se les dan las instrucciones básicas respecto al funcionamiento del mismo. Se les comenta que serán observados por los investigadores directamente pues el laboratorio no dispone de cámara Gesell. Se comenta al sujeto que preste atención al videojuego y se abstenga lo máximo posible de la presencia de los investigadores. El sujeto comienza a jugar y los investigadores se sitúan a su lado.

\subsection{Instrumentos}

Los instrumentos utilizados han sido: la (a) observación, mediante la cual se pretenden percibir microexpresiones propias del lenguaje no verbal señaladas por Ekman, Friesen y Ellsworth (2013) que muestran matices emocionales ante situaciones determinadas; pudiendo identificar paralelismos entre lo que ocurre tras la pantalla como en el propio jugador. En éste sentido, tal y como señalan Mínguez y Fuentes (2004) éste tipo de técnica permite mantener al individuo estudiado en un contexto adecuado y así no modificar sus posibles reacciones; siendo de especial interés las emociones que se manifiesten durante el tiempo de juego sean lo más precisas posibles. Y la (b) entrevista para obtener un registro oral tanto de las emociones percibidas como de los atributos y habilidades que auto-perciben que se trabajan con el videojuego. Los investigadores observarán y tomarán registro de las microexpresiones de los participantes. Además de la toma de notas de las emociones experimentadas durante el proceso de juego, las cuáles se obtendrán mediante preguntas como: ¿Cómo te sientes? ¿Crees haber hecho lo correcto? ¿̇Esta acción te perjudica/beneficia? ¿̇́sta acción perjudica/beneficia al grupo? ¿Cuál crees que es la meta del juego? ¿Por qué has realizado dicha acción? ¿Qué crees que están aprendiendo con esto? (c) registro de observación en el que se incluyan las microexpresiones faciales observadas, la respuesta 
a las preguntas formuladas y el vínculo manifestado por los individuos estudiados respecto a las habilidades y destrezas docentes del siglo XXI.

\subsection{Técnica de Análisis}

Para el análisis de la información se ha utilizado un manual de detección de microexpresiones y emociones (Ekman, Friesen y Ellsworth, 2013) y el programa de análisis cualitativo NVivo en su versión 11.

\section{Resultados}

Tras llevar a cabo la experiencia se ha realizado una tabla con las emociones halladas y un mapa de relaciones con los aprendizajes que se obtienen con los videojuegos.

El análisis de las emociones se basa en las propuestas por Bisquerra y Punset (2015), las cuáles han sido categorizadas según las emociones principales o afines halladas. La recopilación de información se llevó a cabo durante el proceso de juego, en ellas se muestran las emociones que el jugador percibe al jugar, por lo tanto, son subjetivas y vinculadas a la experiencia, así como a las estrategias de resolución de problemas de los sujetos investigados.

Los sujetos investigados destacas las siguientes emociones:

Tabla 1. Tabla de emociones destacadas en los videojuegos analizados.

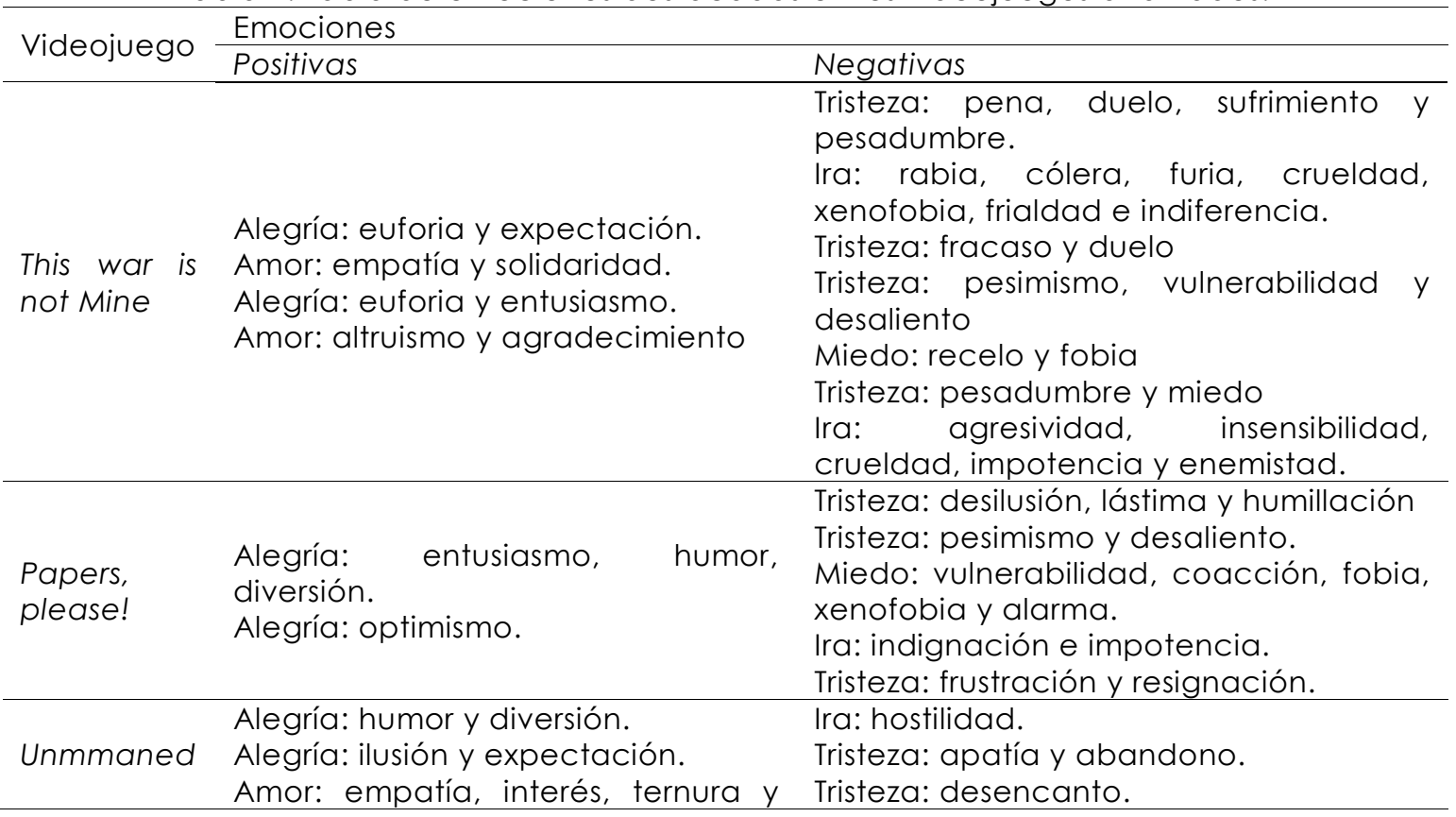




\begin{tabular}{lll}
\hline \multirow{2}{*}{ Videojuego } & Emociones & \\
\cline { 2 - 3 } & Positivas & Negativas \\
\hline & amabilidad. & Tristeza: pesadumbre, duelo. \\
& Alegría: complicidad y humor. & Ira: antipatía y animadversión. \\
\hline
\end{tabular}

El análisis de las emociones halladas en el proceso de juego con This War of Mine, Papers, iplease! y Unmanned (Tabla 1) señala como dominantes aquellas referentes a emociones negativas como la tristeza y la Ira; aun siendo un resultado previsible debido a la narrativa y contextos de desarrollo de los juegos podemos señalar que también se han hallado emociones positivas propias del contexto lúdico como son la alegría y el amor, aunque es menor medida.

Respecto a las habilidades y atributos se han tomado aquellas propuestas por Gerstein (2015) como necesarias para el docente actual puesto que le capacitan para tener un desenvolvimiento óptimo con los contextos tecnológico, social y cultural actuales

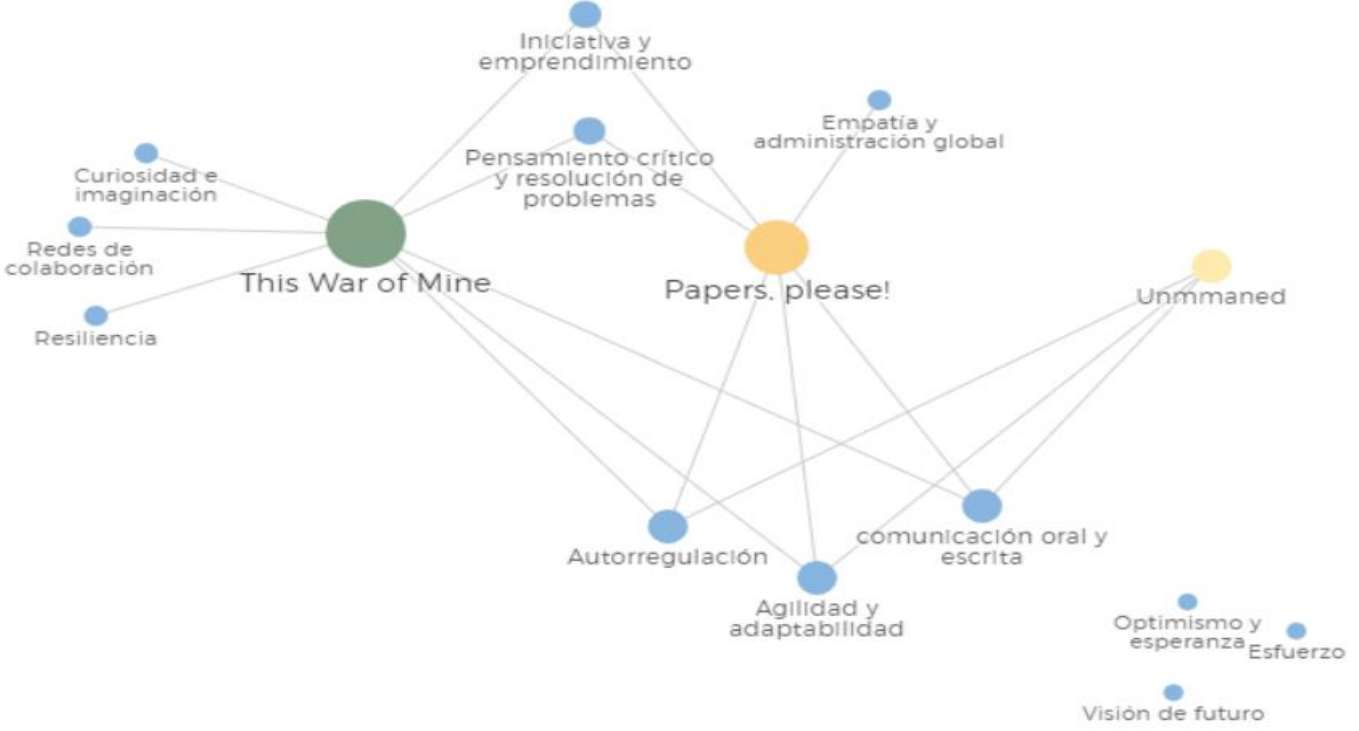

Figura 1. Mapa de relaciones entre los atributos y habilidades del docente del siglo 21 con los videojuegos analizados

Para exponer los resultados se han utilizado nodos que engloben los atributos y habilidades respecto a cada uno de los juegos analizados (Fig. 1). Se va a proceder a realizar una descripción pormenorizada de las relaciones establecidas entre los juegos y dichos atributos y habilidades presentando la vinculación de éstas con cada uno de ellos (Tabla 2). 
Percepción de habilidades docentes a través de las emociones mediante el uso de videojuegos de temática social 
Tabla 2. Relación e las actitudes y habilidades con los videojuegos analizados

\begin{tabular}{|c|c|c|c|}
\hline & $\begin{array}{l}\text { This War } \\
\text { of Mine }\end{array}$ & $\begin{array}{l}\text { Papers, } \\
\text { please! }\end{array}$ & Unmanned \\
\hline Comunicación Oral y escrita & $\mathrm{X}$ & $\mathrm{x}$ & $X$ \\
\hline $\begin{array}{l}\text { Pensamiento crítico y resolución de } \\
\text { problemas }\end{array}$ & $x$ & $x$ & \\
\hline Redes de colaboración & $\mathrm{X}$ & & \\
\hline Curiosidad e imaginación & $\mathrm{X}$ & & \\
\hline Iniciativa y emprendimiento & $x$ & $\mathrm{X}$ & \\
\hline Agilidad y adaptabilidad & $x$ & $x$ & $\mathrm{X}$ \\
\hline Optimismo y esperanza & & & \\
\hline Autoregulación & $\mathrm{x}$ & $x$ & $x$ \\
\hline Empatía y administración global & & $\mathrm{X}$ & \\
\hline Resiliencia & $\mathrm{X}$ & & \\
\hline Esfuerzo & & & \\
\hline Visión de Futuro & & & \\
\hline
\end{tabular}

En base a los resultados obtenidos podemos señalar que el referente a la comunicación oral y escrita es necesario para el avance y la consecución de logros dentro de los mismos. Cabe señalar que la narrativa de éstos juegos se basa en la comprensión de los mensajes, el cuál alude a un aprendizaje de la comunicación a través del juego ya sea mediante los diálogos, escenas de transición o instrucciones.

Par agilidad y adaptabilidad cabe señalar que un videojuego es una herramienta actual y sujeta a cambios cada uno tiene una idiosincrasia propia a la que se debe adaptar el individuo; señalando que los videojuegos son herramientas con una curva de aprendizaje relativamente sencilla en la que a los pocos minutos se es capaz de dominarlo con cierta precisión. Y la autorregulación representa el aprendizaje del videojugador ante las emociones, acciones o pensamientos que le suscita el juego. Como se ha señalado anteriormente son juegos con unas temáticas sociales arduas, en ocasiones con escenas que alientan a la reflexión social con temáticas tan delicadas como el asesinato, la violación, la inmigración o la guerra.

Los atributos y habilidades presentes en dos juegos son el Pensamiento crítico y Resolución de problemas e Iniciativa y el emprendimiento, referente a la necesidad de establecer estrategias de supervivencia en base a la toma de decisiones de un modo rápido, el jugador ha de establecer un liderazgo pensando en las consecuencias de cada acción, como se ha señalado con anterioridad experimentar en un 
contexto virtual permite una toma de decisiones más elaborada cuando se extrapola a la realidad cotidiana.

Para las relacionadas con un solo juego en primer lugar se han tenido en cuenta aquellas vinculadas con This War of Mine, las cuáles son: Curiosidad e imaginación, Redes de colaboración y Resiliencia. A nivel general se puede señalar que al ser un mundo que se genera aleatoriamente cada vez que se juega los deseos de exploración motivan a seguir adelante. Se busca la agrupación de miembros de equipo con aptitudes complementarias que sumen al grupo, es decir crean redes de trabajo para conseguir un progreso máximo.

Aun así, se muestra como las diferentes situaciones perniciosas hacen mella en la mayor parte del grupo induciendo a muchos personajes a la traición o el suicidio. Sin embargo, el jugador se antepone ante éstas y desarrolla al resto de personajes de un modo que pueda combatir las malas experiencias y aprender de ellas para mejorar. El vínculo entre Papers, iplease! y la empatía y administración global se realiza mediante la consideración ideológica del jugador y las del personaje propugnando entre la necesidad de supervivencia o los principios ético-morales del mismo.

Finalmente, existen una serie de actitudes y habilidades no destacadas en ninguno de los juegos como son Optimismo y esperanza, esfuerzo y visión de futuro. Debido a que la temática de los juegos abraza arcos argumentales tendentes al pesimismo y al derrotismo, éstos se impregnan en la visión del jugador. No se puede ser optimista en un contexto en que la desmotivación y la desesperanza colman el discurso. El esfuerzo se esfuma cuando el jugador conoce los finales del juego, y los comprende como una moralina que muestra que las guerras no se ganan, sino que todos pierden.

\section{Discusión}

A modo de conclusión, cabe señalar que la utilización de videojuegos como elemento formativo ha sido positiva, puesto que durante el estudio se hallaron resultados concernientes a las emociones que emergen durante 
el proceso de juego ayudando a comprender los aprendizajes que se producen en éstos. Atendiendo al objetivo específico Elaborar una lista con las emociones halladas en los videojuegos analizados mediante la clasificación que proponen Bisquerra y Punset (2015) evidenciando el hallazgo de una serie de patrones emocionales vinculados con la interacción que se produce entre el video jugador y el videojuego. Tal y como señala McGonigal (2011) se origina empatía respecto a los actos que se realizan o visualizan durante el transcurso de juego. Y debido a la contextualización de la narrativa relacionada con problemáticas sociales, bélicas y ético-morales se presentan emociones mayormente negativas como son: la tristeza, el miedo y la desesperanza; siendo éstas las esperadas en videojuegos de esta tipología.

Respecto al objetivo específico crear un mapa de los vínculos entre el desarrollo de atributos y habilidades y los videojuegos analizados se ha creado un mapa de relaciones entre los tres juegos y los atributos y habilidades para el docente del siglo XXI de Gerstein (2015). En la relación de los juegos con éstos últimos se puede señalar que las personas investigadas señalan que a medida que el juego es más complejo las capacidades de aprendizaje que se le pueden anexionar son mayores. Destacan, por un lado, This War of Mine con aprendizajes vinculados con la reflexión, la resiliencia o el pensamiento crítico. Por otro lado, Papers, please! destaca respecto al resto por el nivel empático de las historias tanto del personaje principal como la de los inmigrantes que intentan pasar la frontera, las cuáles inducen a plantearse dilemas ético-morales. Sin embargo, con Unmanned no destacan ningún aprendizaje significativo respecto a los otros dos, aun así le parece interesante y creen que se trabaja la autorregulación, la comunicación y la adaptabilidad.

Finalmente, existen una serie de aprendizajes que no han sido vinculados con ningún juego como son Optimismo y esperanza, Esfuerzo y visión de futuro, los cuáles aluden a una perspectiva positiva y a largo plazo en unos videojuegos con un triste, paradigmático y aleccionador final.

Para concluir, el objetivo general Analizar los videojuegos seleccionados indagar sobre los patrones emocionales, habilidades $y$ estrategias propias de los docentes del siglo XXI muestra que éste tipo 
videojuegos logran ser un instrumento didáctico para la concienciación y la formación sobre cierto tipo de conflictos y la perspectiva ante éstos. No obstante, aunque desde éste estudio se aportan posibilidades educativas de interés, es necesario estudios más amplios y profundos para elaborar una metodología que permita la utilización en contextos formales como herramientas complementarias al currículo.

\section{Referencias}

BARR, M. (2017). Video games can develop graduate skills in higher education students: A randomised trial. Computers \& Education, 113, 86-97. doi: https://doi.org/10.1016/j.compedu.2017.05.016

BISQUERRA, R., y PUNSET, E. (2015). Universo de emociones. Valencia: PalauGea.

BRUNING, R. H., SCHRAW, G. J., y Norby, M. M. (2011). Cognitive psychology and instruction (5th ed). Boston: Pearson.

DEL MORAL, M. E., y FERNÁNDEZ, L. C. (2012). Comunidades virtuales de videojugadores: Comportamiento emocional y social en Poupée Girl. RED: Revista de Educación a Distancia, 33, 1-19.

DONDLINGER, M. J. (2007). Educational video game design: A review of the literature. Journal of applied educational technology, 4 (1), 21-31.

EKMAN, P., FRIESEN, W. V., y ELLSWORTH, P. (2013). Emotion in the human face: Guidelines for research and an integration of findings. Elsevier.

GEE, J. P. (2003). What video games have to teach us about learning and literacy. Computers in Entertainment, 1(1), 20. doi: https://doi.org/10.1 145/950566.950595

GERSTEIN, J. (2015). The Other 21st Century Skills: Educator Self-Assessment. Recuperado de https://usergeneratededucation.wordpress.com/2015/01/16/the-other21st-century-skills-educator-self-assessment/

GONZÁLEZ, C. S., y BLANCO, F. (2008). Emociones con videojuegos: incrementando la motivación para el aprendizaje. Teoría de la Educación. Educación y Cultura en la Sociedad de la Información, 9 (3). 
GUERRA, J., y REVUELTA, F. I. (2015). Videojuegos precursores de emociones positivas: propuesta metodológica con Minecraft en el aula hospitalaria. IJERI: International Journal of Educational Research and Innovation, 3, 105-120.

GUERRA, J., y REVUELTA, F. I. (2016). Análisis del conocimiento del código PEGI en la formación inicial del profesorado. RELATEC - Revista Latinoamericana de Tecnología Educativa, 15(1), 87-96. doi: https://doi.org/10.17398/1695-288X.15.1.87

KLIMMT, C., HARTMANN, T., Y FREY, A. (2007). Effectance and Control as Determinants of Video Game Enjoyment. CyberPsychology \& Behavior, 10(6), 845-848. doi: https://doi.org/10.1089/cpb.2007.9942

MARCANO, B. E. (2006). Estimulación emocional de los videojuegos: efectos en el aprendizaje. Teoría de la Educación. Educación y Cultura en la Sociedad de la Información, $7(2)$.

MAYER, J. D., y SALOVEY, P. (1997). What is emotional intelligence. Emotional development and emotional intelligence: Educational implications, 3 (31).

MCGONIGAL, J. (2011). Reality is broken: why games make us better and how they can change the world. New York: Penguin Group.

MíNGUEZ, A., y FUENTES, M. (2004). Cómo hacer una investigación social. Valencia: Tirant lo blanch.

PEDRERA, M. I. \& GONZÁLEZ, A. (2017). Percepción del profesorado en formación inicial sobre la aplicación de un instrumento para evaluar el desarrollo de habilidades con juegos en línea. Revista LifePlay, (6).

REVUELTA, F. I., y GUERRA, J. (2012). ¿QUé aprendo con videojuegos? Una perspectiva de meta-aprendizaje del videojugador. Revista de Educación a Distancia, 33.

SHANBARI, H., y ISSA, R. R. A. (2018). Use of video games to enhance construction management education. International Journal of Construction Management, 1-16. doi: https://doi.org/10.1080/15623599.2017.1423166

SQUIRE, K. D. (2008). Video games and education: Designing learning systems for an interactive age. Educational Technology, 48(2), 17. 
Percepción de habilidades docentes a través de las emociones mediante el uso de videojuegos de temática social

TOST, G., y BOIRA, O. (2015). Vida extra: los videojuegos como no los has visto nunca. Barcelona: Grijalbo. 